\title{
Substance P Mediates Excitatory Interactions between Striatal Projection Neurons
}

\author{
Craig P. Blomeley, Laura A. Kehoe, and Enrico Bracci \\ Faculty of Life Sciences, University of Manchester, Manchester M13 9PT, United Kingdom
}

The striatum is the largest nucleus of the basal ganglia, and is crucially involved in motor control. Striatal projection cells are medium-size spiny neurons (MSNs) and form functional GABAergic synapses with other MSNs through their axon collaterals. A subpopulation of MSNs also release substance P (SP), but its role in MSN-MSN communication is unknown. We studied this issue in rat brain slices, in the presence of antagonists for GABA, acetylcholine, dopamine, and opioid receptors; under these conditions, whole-cell paired recordings from MSNs (located $<100 \mu \mathrm{m}$ apart) revealed that, in 31/137 (23\%) pairs, a burst of five spikes in a MSN caused significant facilitation $(14.2 \pm 8.9 \%)$ of evoked glutamatergic responses in the other MSN. Reciprocal facilitation of glutamatergic responses was present in 4 of these pairs. These facilitatory effects were maximal when spikes preceded glutamatergic responses by $100 \mathrm{~ms}$, and were completely blocked by the NK1 receptor antagonist L-732,138. Furthermore, in 31/57 (54\%) MSNs, a burst of 5 antidromic stimuli delivered to MSN axons in the globus pallidus significantly potentiated glutamatergic responses evoked 250 or $500 \mathrm{~ms}$ later by stimulation of the corpus callosum. These effects were larger at 250 than $500 \mathrm{~ms}$ intervals, were completely blocked by L-732,138, and facilitated spike generation. These data demonstrate that MSNs facilitate glutamatergic inputs to neighboring MSNs through spike-released SP acting on NK1 receptors. The current view that MSNs form inhibitory networks characterized by competitive dynamics will have to be updated to incorporate the fact that groups of MSNs interact in an excitatory manner.

\section{Introduction}

The striatum is the main recipient of cortical and thalamic glutamatergic afferents in the basal ganglia, and plays an essential role in motor control (Alexander and Crutcher, 1990; Graybiel et al., 1994; Albin et al., 1995). Striatal neural circuits process this massively convergent excitatory input to generate the striatal inhibitory output, which in turn controls the activity of the basal ganglia output nuclei. Understanding this input-output transformation is one of the central goals in basal ganglia research (Kincaid et al., 1998; Bar-Gad et al., 2003; Gurney et al., 2004; Redgrave and Gurney, 2006). A prominent anatomical feature of the striatum is the dense local network formed by the axon collaterals of its projection neurons (Wilson and Groves, 1980; Somogyi et al., 1981; Yung et al., 1996) that constitute up to $95 \%$ of the striatal neuronal population (Graveland and DiFiglia, 1985). These cells are medium-sized spiny neurons (MSNs) and release GABA (Bolam et al., 2000; Tepper et al., 2004). Their axon collaterals form synapses with other MSNs (Wilson and Groves, 1980; Bolam and Izzo, 1988). Paired recording experiments have shown that GABAergic synapses between neighboring MSNs are functional, and are found (at least in one direction) in 20-29\% of MSN pairs (Tunstall et al., 2002; Koos et al., 2004; Shindou et al., 2008). GABAergic inhibition between MSNs has been proposed to

Received Nov. 24, 2008; revised March 10, 2009; accepted March 24, 2009.

This study was funded by The Wellcome Trust and the Royal Society. We thank Dr. Jon Turner and Ritchie Smith for their useful comments on this manuscript.

Correspondence should be addressed to Enrico Bracci, Faculty of Life Sciences, University of Manchester, AV Hill Building, Manchester M13 9PT, UK. E-mail: e.bracci@manchester.ac.uk.

DOI:10.1523/JNEUROSCI.6020-08.2009

Copyright $\odot 2009$ Society for Neuroscience $\quad$ 0270-6474/09/294953-11\$15.00/0 underlie recognition and classification of cortical patterns (Beiser and Houk, 1998; Bar-Gad et al., 2003; Wickens et al., 2007).

MSNs belong to two subpopulations: those projecting directly to the basal ganglia output nuclei express substance P (SP), dynorphin, and $\mathrm{D}_{1}$ dopamine receptors, whereas those projecting indirectly to the output nuclei express enkephalin and $D_{2}$ dopamine receptors (Gerfen, 1992; Wang et al., 2006; Gertler et al., 2008). Beside GABA, SP is a potential mediator of synaptic communication between MSNs; in fact, SP-containing terminals form synapses with spines, dendrites, and cell bodies of MSNs belonging to both subpopulations (Bolam and Izzo, 1988; Yung et al., 1996). Immunohistological studies have shown that presynaptic NK1 receptors (for which SP is the main endogenous ligand; Almeida et al., 2004; Baranauskas et al., 1995) are present on glutamatergic terminals in the striatum (Jakab and GoldmanRakic, 1996). Consistent with these anatomical findings, we have recently reported that exogenous SP strongly facilitates evoked glutamatergic responses of MSNs, and that this effect is mediated by presynaptic NK1 and NK3 receptors (Blomeley and Bracci, 2008). These findings raised the possibility that endogenous SP released by MSNs may facilitate the glutamatergic responses of other neighboring MSNs, thus giving rise to excitatory interactions. In the present study we tested this hypothesis using a paired recording protocol we recently developed to study presynaptic interactions (Pakhotin and Bracci, 2007), as well as antidromic activation of MSN axons triggered by stimulation delivered in the globus pallidus (GP) (Guzmán et al., 2003).

\section{Materials and Methods}

Slice preparation. Wistar rats (14-24 d postnatal, both sexes) were killed by cervical dislocation in accordance with the UK Animals Act 1986; 
coronal or parasagittal brain slices (250-300 $\mu \mathrm{m}$ thick) were cut using a vibroslicer (Camden Instruments) and maintained at $25^{\circ} \mathrm{C}$ in oxygenated artificial CSF (ACSF) (in mM: $126 \mathrm{NaCl}, 2.5 \mathrm{KCl}, 1.3 \mathrm{MgCl}_{2}, 1.2$ $\mathrm{NaH}_{2} \mathrm{PO}_{4}, 2.4 \mathrm{CaCl}_{2}, 10$ glucose, and $\left.18 \mathrm{NaHCO}_{3}\right)$. For recordings, slices were submerged, superfused $(2-3 \mathrm{ml} / \mathrm{min})$ at $25^{\circ} \mathrm{C}$, and visualized with a $10 \times$ and $40 \times$ water-immersion objectives (Olympus Optical) using standard infrared and differential interference contrast microscopy.

Whole-cell recordings. Whole-cell recordings from single MSNs, or from pairs of MSNs, were performed with $1.5 \mathrm{~mm}$ external diameter borosilicate pipettes, filled with intracellular solution (in mM: 125 K-gluconate, $15 \mathrm{KCl}, 0.04 \mathrm{EGTA}, 12 \mathrm{HEPES}, 2 \mathrm{MgCl}_{2}, 4 \mathrm{Na}_{2} \mathrm{ATP}$, and 0.4 $\mathrm{Na}_{2} \mathrm{GTP}$, adjusted to $\mathrm{pH} 7.3$ with $\mathrm{KOH}$ ). With this solution, pipette resistance was 3-4 M $\Omega$. Recordings in current-clamp mode were performed with two different bridge amplifiers (AxoClamp 2B, Molecular Devices; and BA-1S, NPI). Recordings in voltage-clamp mode were made using the AxoClamp 2B in continuous single-electrode mode with uncompensated series resistance. Data were acquired at $10 \mathrm{kHz}$ using Signal software and a micro1401 data acquisition unit (Cambridge Electronic Design). Input resistance was monitored under current-clamp conditions with small negative current injections ( $500 \mathrm{~ms}$ to $1 \mathrm{~s} ; 15-20 \mathrm{pA}$ ).

In paired recording experiments, the distance between two MSNs was measured as the minimal distance between the somata of the cells as visible with the optics used. This is less than the distance between the centers of the cells, which was not unequivocally defined under the present conditions. Input resistance in current-clamp experiments was measured with small current injections at resting membrane potential. In the presence of a treatment that caused depolarizations, resistance was measured while the cells were briefly repolarized to control level.

Drugs. Drugs were bath-applied through the superfusion system. NBQX and AP-5 were obtained from Sigma-Aldrich and atropine sulfate, naloxone hydrochloride, S-(-)-sulpiride, SCH 23390 hydrochloride, CGP 52432, bicuculline, L-732,138, and tubocurarine chloride from Tocris.

Evoked synaptic responses. Glutamatergic responses were recorded in current-clamp mode as EPSPs or in voltage-clamp as EPSCs. These responses were evoked with a monopolar stimulating electrode, consisting of a patch micropipette filled with ACSF, placed either in the corpus callosum (CC), between the cortex and the striatum, or in the striatum just below $(<50 \mu \mathrm{m})$ the white matter, both in coronal and sagittal slices; in both cases, this procedure aimed at activating preferentially corticostriatal fibers. The resistance of the micropipettes used for stimulation was $0.3-1 \mathrm{M} \Omega$. Stimulation amplitude was $10-100 \mathrm{~V}$, and its duration was 0.01-0.1 ms. All the experiments were performed in the continuous presence of antagonists of the following receptors: $\mathrm{GABA}_{\mathrm{A}}$ (bicuculline, $10 \mu \mathrm{M}) ; \mathrm{GABA}_{\mathrm{B}}$ (CGP 52432, $2 \mu \mathrm{M}$ ); opioid (naloxone hydrochloride, 10 $\mu \mathrm{M}$ ); dopamine $\mathrm{D}_{1}\left(\mathrm{SCH} 23390\right.$ hydrochloride, $10 \mu \mathrm{M}$ ); dopamine $\mathrm{D}_{2}$ [S-(-)-sulpiride, $3 \mu \mathrm{M}]$, muscarinic (atropine sulfate, $25 \mu \mathrm{M}$ ), and nicotinic (tubocurarine chloride, $10 \mu \mathrm{M}$ ). Under these conditions, evoked responses, recorded under either current-clamp or voltage-clamp conditions, were completely abolished by coapplication of the ionotropic glutamate receptor antagonists 2,3-dihydroxy-6-nitro-7-sulfonyl benzo[f] quinoxaline (NBQX) $(10 \mu \mathrm{M})$ and AP-5 $(10 \mu \mathrm{M})(n=20)$. A single electrical stimulus was applied every $10 \mathrm{~s}$. This interval was chosen because, after the first 10 stimuli, the EPSC amplitude did not display a statistically significant trend of variation as a function of time (ANOVA regression analysis always yielded significance levels $>0.05$ ). On the other hand, the first 3-10 responses were often significantly larger than the following ones. Therefore the first 10 responses were always discarded from analysis. Antidromic stimulation of MSN axons was achieved in sagittal slices using a second stimulating electrode (similar to the one used to stimulate corticostriatal fibers) placed in the GP, as illustrated in Figure $5 A$. GP stimulation also elicited glutamatergic responses in MSNs; In some experiments, we tried to reduce the duration of these responses, which was defined as the time elapsed after the last GP stimulus before the evoked EPSP subsided to $10 \%$ of its peak value.

Spike-evoked response sequences. Paired recordings were performed to investigate whether action potentials in a MSN could affect the glutamatergic responses of another MSN located nearby. The two simultaneously recorded MSNs were arbitrarily named MSN1 and MSN2. Spikes were elicited in MSNs under current-clamp conditions by using short ( $3 \mathrm{~ms}$ ) but relatively large (1.0-1.5 nA) current injections, separated by $10 \mathrm{~ms}$ intervals. This procedure allowed us to trigger bursts of spikes repetitively (at $77 \mathrm{~Hz}$ ) with high temporal precision. An example of this procedure is shown in Figure $1 C$.

In these experiments, a single glutamatergic response was evoked every $10 \mathrm{~s}$ by stimulating the corticostriatal fibers. Each cortical stimulus was sequentially preceded by (1) no spikes in MSN1 or MSN2 (control); (2) a burst of spikes in MSN1; and (3) a burst of spikes in MSN2. This threestep sequence is illustrated in Figure $1 D$ and was repeated, without interruptions, 60-150 times for each pharmacological condition in each experiment. Data for each condition $(1,2$, or 3$)$ and each pharmacological condition were grouped after the experiments for averaging and statistical analysis. The continuous repetition of the three protocols ensured that any slow change over time in the recording conditions would affect each condition equally. However, we continuously monitored the quality of the MSN recordings, and only the experiments in which the membrane potential and the input resistance of both cells did not change by $>5 \%$ during the whole experiment were accepted for analysis. The interval between the spikes in one MSN and the glutamatergic response of the other cell was measured from the start of the current injection that elicited the first spike of a burst to the artifact of the electrical stimulation.

Statistical analysis. Values are expressed as mean $\pm \mathrm{SD}$, and statistical comparisons between two groups were made using Mann-Whitney test for unpaired data. We chose this nonparametric test as no assumption is needed about normality of data distribution. Statistical comparison of three groups of data was performed with Kruskal-Wallis test. Statistical comparison of the frequency of action potential generation in the absence or presence of preceding antidromic MSN stimulation was performed with McNemar's test for paired data. For this purpose, each stimulation trial without preceding antidromic MSN stimulation was paired with the subsequent one (that was preceded by antidromic stimulation).

Two populations of data points were considered to be significantly different if $p<0.05$. Statistical tests and cumulative frequency plots were implemented with MATLAB software (The MathWorks).

\section{Results}

Current- and voltage-clamp whole-cell recordings were obtained from 348 MSNs, identified by their distinctive electrophysiological properties (Kita et al., 1984; Nisenbaum and Wilson, 1995; Bracci et al., 2004). These properties included resting membrane potentials more negative than $-75 \mathrm{mV}$; strong inward rectification giving rise to asymmetric responses to negative and positive current injections of the same magnitude; slow depolarizing ramps during subthreshold depolarizations; and delayed appearance of action potentials during suprathreshold depolarizing steps. The average resting membrane potential was $-76 \pm 7 \mathrm{mV}$. The average input resistance was $214 \pm 75 \mathrm{M} \Omega$. Typical MSN responses are illustrated in Figure $1 B$.

To investigate the effects of synaptically released SP on glutamatergic input to MSNs, we performed paired recordings from MSNs located $<100 \mu \mathrm{m}$ apart in coronal brain slices. All recorded cells were $>500 \mu \mathrm{m}$ from the stimulating electrode. The typical positioning of the recording and stimulating electrodes is shown in Figure $1 A$. The protocol used to study interactions between MSNs is described in the Materials and Methods, and illustrated in Figure $1 C$. Bursts of precisely timed action potentials were elicited (at $77 \mathrm{~Hz}$ ) in each of the two recorded MSNs with brief current injections, as described in the Materials and Methods and illustrated in Figure $1 D$; in the experiment of Figure $1 D$, a burst of five spikes was elicited in a MSN every $10 \mathrm{~s}$. Superimposition of 150 consecutive trials shows that the temporal scattering of individual spike peaks was $<1 \mathrm{~ms}$. To isolate pharmacologically the evoked glutamatergic responses, and to limit the activation of other types of presynaptic receptors, all the experi- 
A
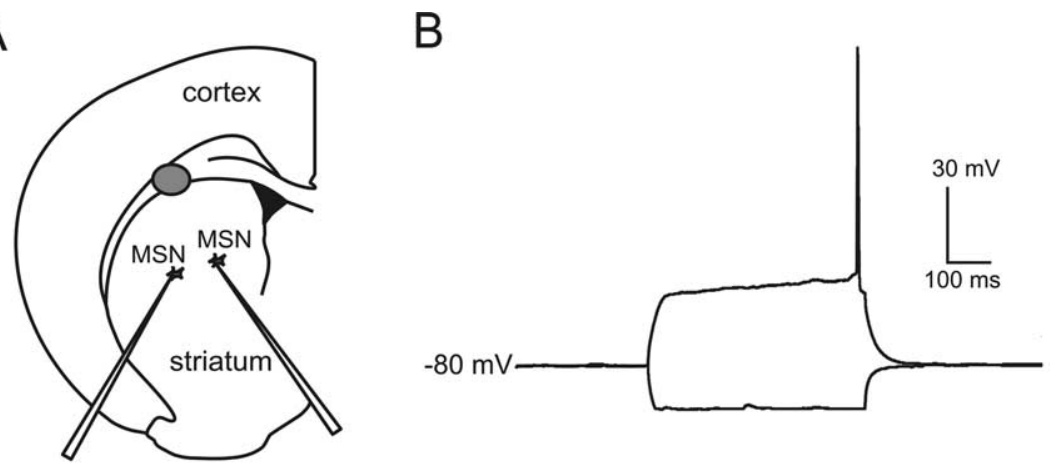

C

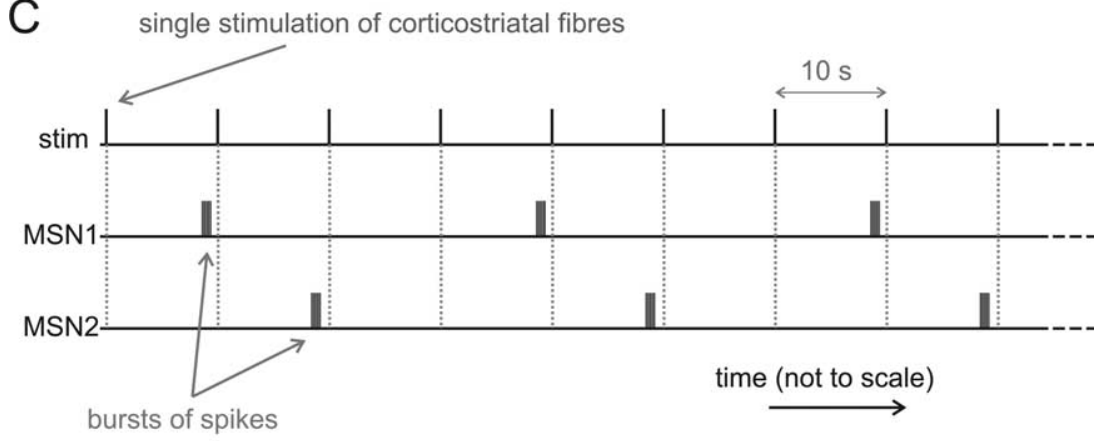

D

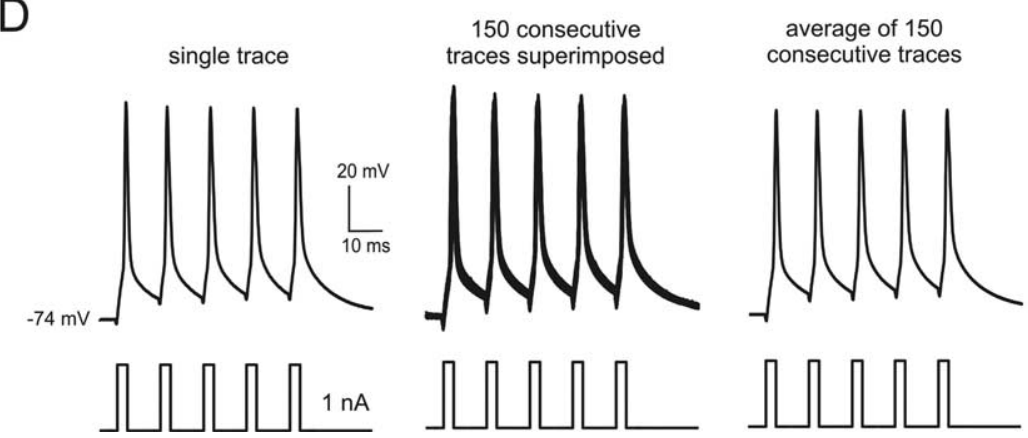

Figure 1. Experimental configuration for paired recording experiments. $\boldsymbol{A}$, Typical positioning of the electrodes used for whole-cell paired recordings from MSNs (located $<100 \mu \mathrm{m}$ apart), and of the stimulating electrode (gray area) used to activate corticostriatal fibers end evoke glutamatergic responses in MSNs in coronal brain slices. $\boldsymbol{B}$, Typical responses of MSNs to positive and negative current injections $( \pm 200 \mathrm{pA})$. The positive injection was just large enough to elicit one spike. Note the depolarizing ramp causing delayed firing, and the smaller extent of responses to negative steps, indicative of inward rectification. C, Graphical illustration of the protocol used to investigate the effects of spikes in each MSN on the glutamatergic responses of the other MSN. For sake of clarity, time is not represented to scale, as the intervals between single stimuli to corticostriatal fibers (10 s) have been compressed with respect to the duration of the bursts of spikes and the interval between such bursts and the stimulation of the corticostriatal fibers. D, Bursts of precisely timed action potentials were evoked in MSNs using 3 ms current injections separated by $10 \mathrm{~ms}$ intervals; this protocol was repeated every $10 \mathrm{~s}$. In this experiment, 150 bursts (each comprising 5 spikes) were evoked. Superimposition of 150 consecutive traces (middle) shows that there was little scattering $(<1 \mathrm{~ms})$ in the action potential peak time; the average of the 150 traces is shown on the right.

ments were performed in the presence of $\mathrm{GABA}_{\mathrm{A}}, \mathrm{GABA}_{\mathrm{B}}$, opioid, dopamine $\left(\mathrm{D}_{1}\right.$ and $\left.\mathrm{D}_{2}\right)$, nicotinic, and muscarinic receptor antagonists (see Materials and Methods for details). It should be noted that direct GABAergic connections between MSNs (Tunstall et al., 2002; Tepper et al., 2008) were pharmacologically blocked throughout these experiments.

\section{Effects of MSN action potentials on MSN} membrane properties

In a previous study, we showed that exogenous SP can directly depolarize MSNs by activating an inward rectifying chloride cur- rent (Blomeley and Bracci, 2008). We therefore investigated if spikes in a MSN could evoke depolarizing postsynaptic responses in a neighboring MSN. However, despite recording from 137 pairs under current-clamp conditions, we did not observe any postsynaptic effect in MSNs that were time locked to the spikes of a neighboring MSN. Examples of this lack of direct effects can be seen in the experiments illustrated in Figures $2 \mathrm{~A}, 3 \mathrm{~A}$, and $4 \mathrm{~A}$. We also failed to observe slower depolarizing effects that could have resulted from repetitive burst firing in MSNs. We conclude that direct SP-mediated synaptic communication between MSNs was not significant under these conditions.

\section{Effects of MSN action potentials on MSN glutamatergic responses}

In these 137 MSN pairs, we used the protocol illustrated in Figure $1 C$ to test whether spikes in one MSN affected the glutamatergic responses of the other MSN. Preliminary experiments showed that bursts of five spikes, with the first spike preceding the stimulation of afferent fibers by $100 \mathrm{~ms}$, were particularly effective in facilitating glutamatergic responses. Therefore, we used this temporal sequence to test for the presence of interactions in MSN-MSN pairs. With this protocol, we found significant facilitation of glutamatergic responses at least in one direction (i.e., a burst of spikes in one MSN significantly increased the amplitude of the evoked EPSPs in the other MSN) in 31/137 pairs (23\%). In 4 of these 31 pairs, the interactions were bidirectional, i.e., spikes in each MSN caused significant facilitation of evoked EPSPs in the other MSN. A representative example of these bidirectional interactions is shown in Figure 2, where each trace in $A$ and $B$ is the average of 100 consecutive traces. The cumulative distributions of the evoked EPSP amplitude for each MSNs, in the absence or presence of spikes in the other MSN, are shown in Figure 2C; the presence of a burst of spikes in MSN1 caused a rightward shift of the distribution of MSN2 responses (with respect to the control one, in which no spikes were present), and vice versa.

In the remaining $106 / 137$ pairs, the presence of preceding spikes in either MSNs did not significantly affect the evoked responses of the other MSN; spike-induced inhibition of the glutamatergic responses was never observed under the pharmacological conditions of this study. The overall distribution of the effects of a burst of spikes in a MSN on the responses of the other MSN is illustrated by the histogram of Figure $2 D$. The white part of each column indicates cases in which no significant effects were found, while the black part indicates cases in which significant facilitation was present. In the cases in which significant facilitation was found, the spikeinduced increase in EPSP amplitude was on average 14.2 \pm 8.9\%. 
The distribution of these effects is enlarged in the histogram of Figure $2 E$.

Of the 137 MSNs pairs, in which modulation of glutamatergic responses was studied, 74 were recorded in slices from animals aged P21-P24; in these 74 experiments, significant facilitation (12.5 士 $3.7 \%$ ) of glutamatergic input was found in 14 cases $(19 \%)$. This percentage is similar to the one observed in the whole population, showing that, within the age range tested, these effects were not prevalent in preweaning animals.

To test whether spike-induced facilitation of glutamatergic responses was due to SP release, we bath-applied the NK1 receptor antagonist L-732,138 in 11 paired recording experiments in which significant effects had been observed in control solution. As previously reported (Blomeley and Bracci, 2008), in 18 of these 22 MSNs application of L-732,138 per se caused a significant $(p<0.01)$ decrease in the amplitude of control glutamatergic responses (i.e., not preceded by spikes in the other MSN). Furthermore, in all 11 pairs L-732,138 fully blocked the ability of MSN spikes to affect the glutamatergic responses in the other MSN; an example of these effects is shown Figure 3. We concluded that the facilitatory effects of MSN spikes on glutamatergic responses were entirely mediated by NK1 receptors.

We then investigated how these facilitatory effects depended on the interval between the spikes and the evoked glutamatergic response. In these experiments the protocol was similar to that illustrated in Figure $1 C$, but the interval between the first spike of a burst and the stimulation of afferent fibers was sequentially set to 50,100, and $200 \mathrm{~ms}$ in each MSN. In five cases in which significant effects were observed for $100 \mathrm{~ms}$ intervals, no significant effects were observed for $50 \mathrm{~ms}$ intervals; when the interval was $200 \mathrm{~ms}$, significant facilitation was still present (although it was significantly smaller than for $100 \mathrm{~ms}$ interval; $p<0.05)$ in $2 / 5$ pairs, while no facilitation was present in the other 3 pairs. A representative example of these experiments is shown in Figure $4 \mathrm{~A}$. In this case, spikes in MSN2 significantly increased the amplitude of the evoked EPSP in MSN1 at $100 \mathrm{~ms}$, but no significant effects were observed at 50 or $200 \mathrm{~ms}$. The plot of Figure $4 B$ shows the average results obtained in each of the five experiments. Overall average results are shown in Figure 4C. These data showed that the effects of MSN spikes depended quite steeply on the interval from the EPSP, with maximal facilitatory effects occurring 100 ms after the first spike of a burst.
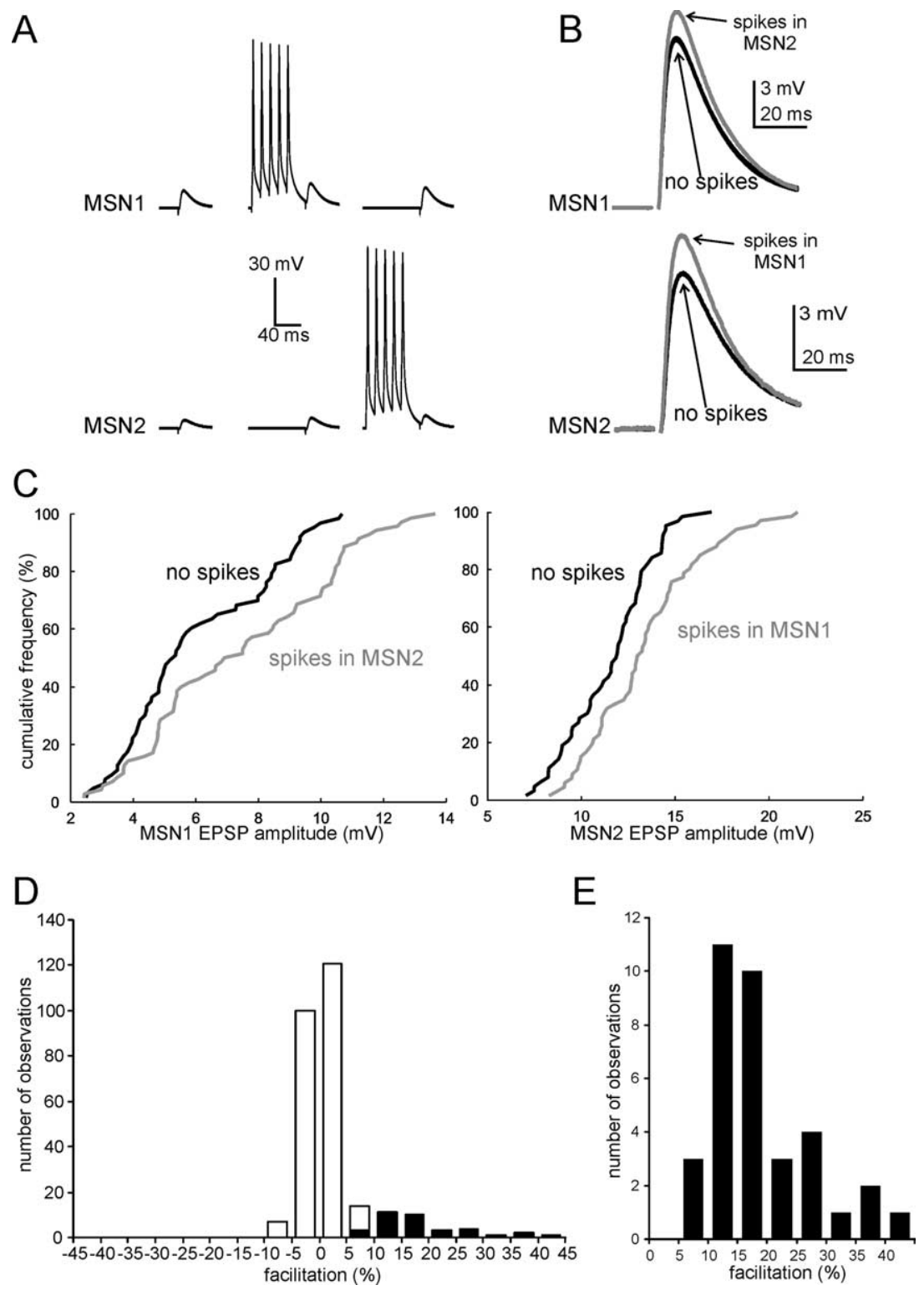

Figure 2. Action potentials in MSNs facilitate glutamatergic responses of neighboring MSNs. $\boldsymbol{A}$, Average responses of two simultaneously recorded MSNs to stimulation of corticostriatal fibers in the absence of preceding spikes (left); in the presence of a preceding burst of five spikes in MSN1 (middle); and in the presence of a preceding burst of five spikes in MSN2 (right). The first spike of a burst in MSN1 or MSN2 preceded the stimulation of corticostriatal fibers by $100 \mathrm{~ms}$. These three protocols were applied sequentially at 10 s intervals (each protocol was applied 100 times in this experiment). $\boldsymbol{B}$, Enlargement of average glutamatergic responses in MSN1 and MSN2 (same experiments as in $\boldsymbol{A}$ ) in the absence (black trace) or the presence (gray trace) of preceding spikes in the other MSN. In this experiment, spikes in each MSN significantly $(p<0.001)$ facilitated the responses of the neighboring MSN. C, Cumulative distribution of EPSP amplitude in the same MSNs of $\boldsymbol{A}$ and $\boldsymbol{B}$, in the absence (black line) or presence (gray line) of preceding action potentials in the other MSN. In both cases, a rightward shift of the distribution in the presence of spikes in the other cell is clearly visible. $D$, Overall distribution of the effects of a burst of five spikes in a neighboring MSN on the amplitude of glutamatergic responses evoked by stimulation of corticostriatal fibers. Data are from 137 paired recording experiments (for each of which effects in both directions were considered). The white part of each column represents cases in which no significant effects of the spikes were observed; the black part represents cases in which significant $(p<0.05)$ facilitation was present; note that significant inhibition of evoked responses was never observed under the conditions of this study. $\boldsymbol{E}$, An enlargement of the distribution of the effects for the cases in which significant facilitation was present.

\section{Effects of MSN antidromic stimulation on MSN glutamatergic responses}

The paired recording experiments provided useful information on the effects of spikes in a single MSN on the glutamatergic responses of another MSN. Using parasagittal brain slices, it is possible to evoke antidromic spikes in a population of MSNs. Stimulations delivered in the GP are expected to trigger anti- 

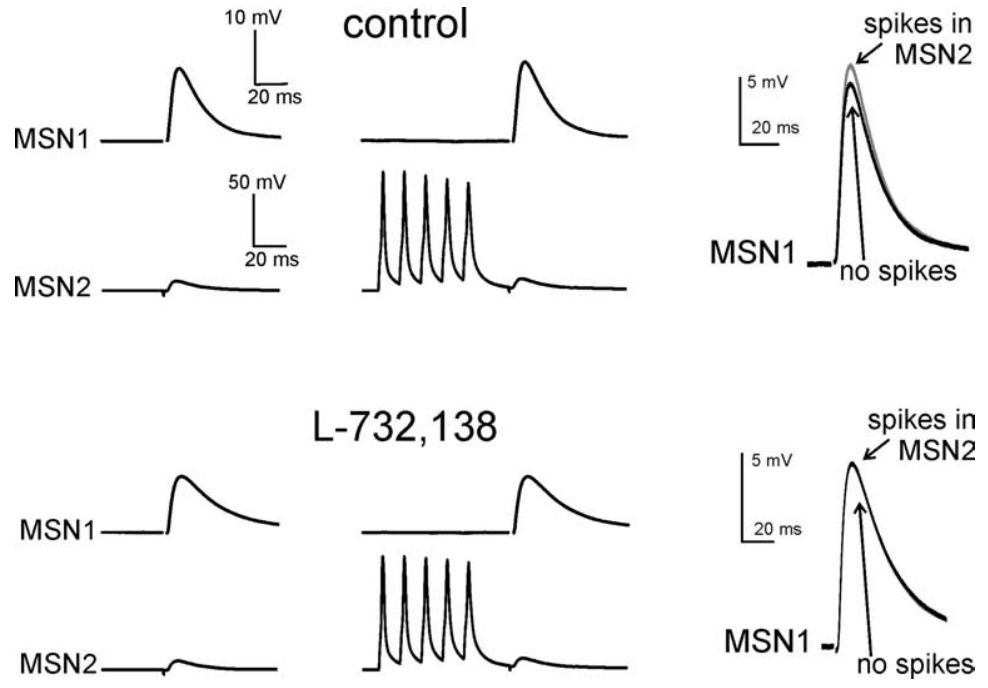

Figure 3. The facilitatory effects of MSN spikes on glutamatergic responses are mediated by NK1 receptors. In this representative example, 5 spikes in MSN2 significantly $(p<0.05)$ increased EPSP amplitude in MSN1. Traces are averages of 100 consecutive presentations for each protocol. Superimposed traces on the right show the average evoked EPSP in MSN1 with (gray) and without (black) preceding spikes in MSN2 for each pharmacological condition. Subsequent bath application of the NK1 receptor antagonist L-732,138 (5 $\mu \mathrm{M})$ fully blocked the ability of MSN2 spikes to facilitate MSN1 evoked glutamatergic responses. L-732,138 also significantly decreased the amplitude of basal evoked glutamatergic responses.

dromic spikes in a large population of MSNs, as the axons of both striatonigral and striatopallidal neurons pass through, or terminate in, this region. This technique has been recently introduced and successfully used to study the effects of synchronous firing of MSNs (Guzmán et al., 2003; Tecuapetla et al., 2005). We used a similar experimental configuration to explore whether spikes in a population of MSNs could affect the evoked glutamatergic responses of a MSN to a larger extent, and for a longer period than those of a single MSN. In these experiments, a MSN was recorded in whole-cell configuration, while the axons of MSNs were stimulated antidromically in the GP. A second stimulating electrode was placed in the white matter of the corpus callosum (CC) between the cortex and the striatum, or in the adjacent striatal area, and was used to activate preferentially corticostriatal fibers. The position of the two stimulating electrodes in these experiments is illustrated in Figure 5A. All recorded MSNs were $>500 \mu \mathrm{m}$ from either stimulating electrode. These experiments were performed in the presence of the same receptor antagonists used for the paired recording experiments. The intensity of the GP stimulation was adjusted to $60-70 \%$ of the minimum intensity eliciting antidromic spikes in the recorded MSN; this was chosen as a trade-off between the need to activate a large number of MSNs, and that to avoid antidromic spikes in the recorded MSN (which would have complicated the interpretation of the results), and to limit the extent of the glutamatergic responses which were evoked by GP stimulation; these responses were presumably due to antidromic activation of corticostriatal fibers directed toward the brainstem (Wilson, 1987; Jaeger et al., 1994), and orthodromic and/or antidromic activation of thalamostriatal fibers (Wilson et al., 1983). Examples of GP-evoked responses can be seen in Figures $5 C, 6 A$, and $7 A$. GP-evoked glutamatergic responses were not of interest in the present study, but it was not possible to abolish them, as the functional integrity of ionotropic glutamate receptors was vital for these experiments. The relatively long duration of such GP-evoked responses limited our ability to investigate the effects of GP stimuli on corticostriatal response evoked at intervals as short as those used in paired re- cordings. Nevertheless, we found that that GP stimulation could strongly facilitate CC-evoked responses of MSNs for intervals as long as $500 \mathrm{~ms}$. A protocol similar to that used for paired recordings was implemented; 100-600 single glutamatergic responses were evoked by CC stimulation every $10 \mathrm{~s}$. Every other time, CC stimulation was preceded by a burst of five GP stimuli (at $100 \mathrm{~Hz}$ ). The CC-evoked responses with or without preceding GP stimulation, were then grouped for statistical analysis. Using a $500 \mathrm{~ms}$ interval (defined as the temporal distance between the first GP stimulus and the CC stimulus), significant facilitatory effects of preceding GP stimulation on CC-evoked responses were found in 18/41 (44\%) MSNs. In these cells, average facilitation was $22 \pm 9 \%$. Statistical tests performed on the EPSP responses with or without preceding GP stimulation, yielded $p<0.05$ in 6 , and $p<$ 0.001 in the remaining 12 cases. An example of the facilitation of CC-evoked responses caused by preceding GP stimulation is shown in Figure $5 C$ (top traces). In the remaining $23 \mathrm{MSNs}$, GP stimulation did not cause significant changes in the amplitude of corticostriatal responses. Of these 41 experiments, 19 were performed in slices from animals aged P21$\mathrm{P} 24$; in this subgroup, significant facilitation $(17 \pm 4 \%)$ of CCevoked responses was found in $11 / 19$ cases (58\%). Thus, the facilitatory effects of GP stimulation were found with similar frequency in MSNs of preweaning and postweaning animals in the age range tested.

In 11 experiments in which significant facilitation was observed, we subsequently applied L-732,138. In all cases, this antagonist fully blocked the effects of GP stimulation on CC-evoked responses, as shown in the example of Figure $5 C$ (bottom traces). Similar to what observed in paired recording experiments, L-732,138 also produced a significant $(p<0.001)$ decrease in basal (i.e., not preceded by spikes) CC-evoked responses amplitude in all cases tested. Figure $5 D$ shows the cumulative distribution of cortically evoked EPSP amplitudes for the experiments of Figure 5C, before (left) and after (right) application of L-732,138. In the absence of L-732,138, preceding GP stimulation caused a clear rightward shift in the distribution, while this is not the case in the presence of the antagonist. We concluded that, as in the case of the facilitation caused by action potentials in a single MSN, facilitation of glutamatergic responses caused by preceding GP stimulation was due to activation of NK1 receptors.

In another series of experiments, we compared the effects of five GP stimuli (at $100 \mathrm{~Hz}$ ), with those of a single GP stimulus. This was accomplished by delivering a single CC stimulus every $10 \mathrm{~s}$, preceded sequentially by (1) no GP stimulation; (2) a single GP stimulus (500 ms interval); and (3) five GP stimuli (500 ms interval). In 6 experiments in which a burst of five GP stimuli (preceding CC stimulation by $500 \mathrm{~ms}$ ) caused significant $(p<$ 0.001 ) facilitation of CC-evoked responses in MSNs, we found that the facilitatory effects of a single GP stimulus were either absent (in $3 / 6$ cases), or still present $(p<0.05)$, but significantly $(p<0.05)$ smaller than that caused by 5 stimuli. An example of these experiments is shown in Figure $6 A$, while the average effects for these 6 experiments are shown in Figure $6 B$. We concluded 
A

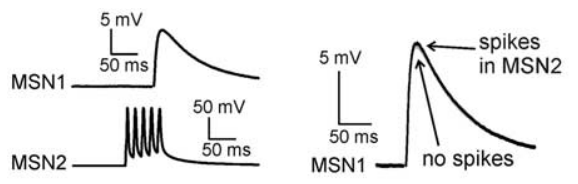

$50 \mathrm{~ms}$ interval

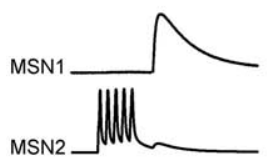

$100 \mathrm{~ms}$ interval

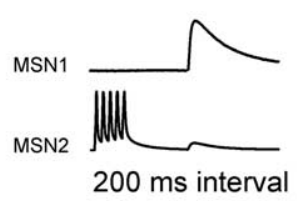

B

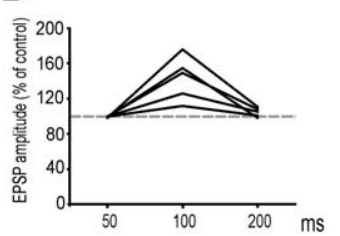

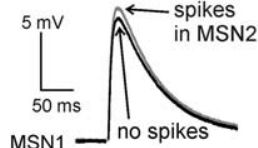

MSN1 _ no spikes

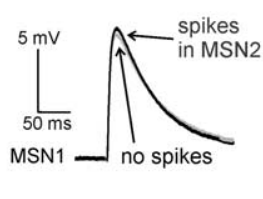

C

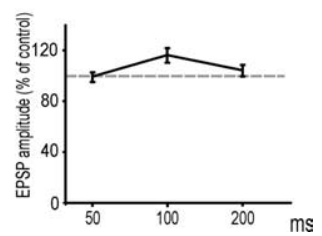

Figure 4. The facilitatory effects of MSN spikes on glutamatergic responses are maximal at $100 \mathrm{~ms}$ intervals. $\boldsymbol{A}$, In this experiment, a burst of 5 spikes in MSN2 significantly $(p<0.05)$ increased EPSP amplitude in MSN1 when the interval between the first spike and the glutamatergic response was $100 \mathrm{~ms}$; however, no significant facilitation was present when this interval was 50 or $200 \mathrm{~ms}$. Traces are averages of 100 consecutive presentations for each protocol. Superimposed traces on the right show the average evoked EPSP in MSN1 with (gray) and without (black) preceding spikes in MSN2. $\boldsymbol{B}$, Effects of a burst of five spikes in a MSN on the evoked glutamatergic responses of a neighboring MSN, for intervals of 50, 100, and $200 \mathrm{~ms}$. Data are from five paired recording experiments in which significant facilitatory effects were observed (in one direction) for intervals of $100 \mathrm{~ms}$. Each point represents the average effect of the spikes for a given interval; data from the same experiment are connected by lines. In all cases, no significant facilitation was present at 50 ms intervals. In two of five cases there was still significant $(p<0.05$ ) facilitation at $200 \mathrm{~ms}$, although this was always smaller than at $100 \mathrm{~ms}$. $\boldsymbol{C}$, Average effects for the five experiments of $\boldsymbol{B}$. Error bars represent SDs.

that a burst of five antidromic spikes in MSNs was more effective than a single antidromic spike in facilitating glutamatergic responses on MSNs

Given the time course of the effects observed with paired recording experiments, it was of interest to investigate the effects of GP stimulation at shorter intervals from the CC stimulation. As mentioned above, this possibility was limited by the fact that GP stimuli evoked relatively slow glutamatergic responses, which would overlap with CC-evoked ones, complicating the interpretation of the results. Therefore, we tried to reduce the duration of GP-evoked responses with bath application of the NMDA receptor antagonists APV $(10 \mu \mathrm{M})$, and MPEP hydrochloride $(1 \mu \mathrm{M})$ an antagonist of metabotropic glutamatergic receptor mGluR5, which is strongly expressed by MSNs (Testa et al., 1995) However, both APV $(n=5)$ and APV $+\operatorname{MPEP}(n=5)$ failed to significantly affect the duration of GP-evoked responses.

As expected, GP-evoked EPSCs recorded under voltageclamp conditions were generally shorter than GP-evoked EPSP recorded under current clamp conditions. Therefore, voltageclamp recordings were used, as these often allowed us to test intervals as short as $250 \mathrm{~ms}$. In a series of experiments, the effects of 5 GP stimuli on cortical responses at 250 and $500 \mathrm{~ms}$ were
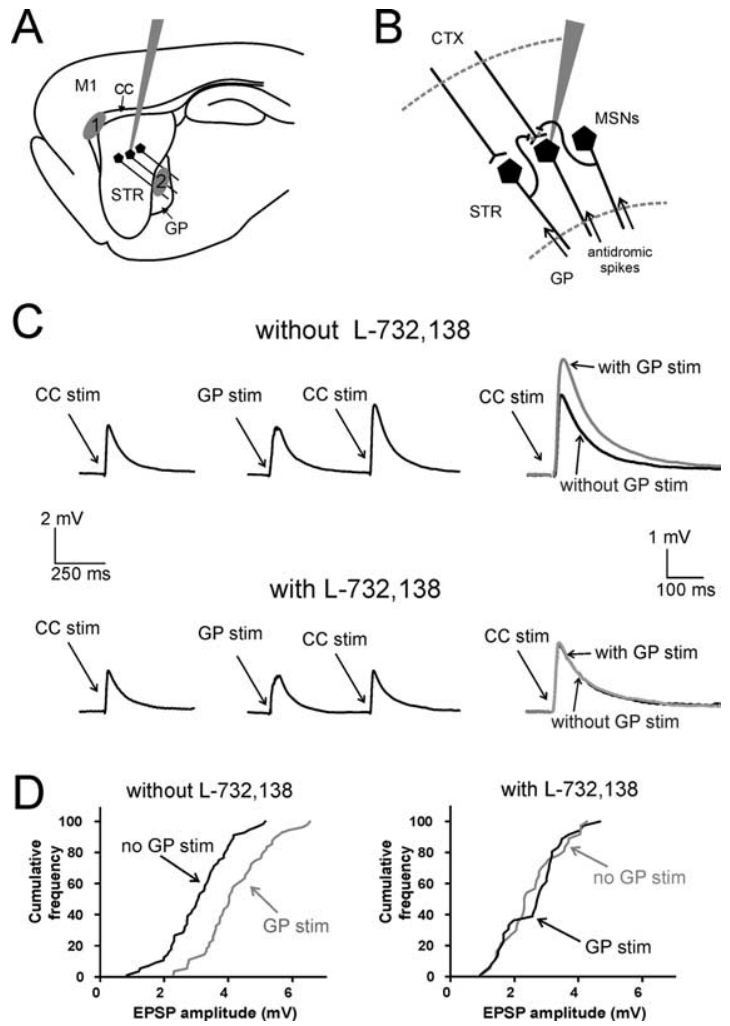

Figure 5. Antidromic stimulation of a population of MSNs facilitates evoked corticostriatal glutamatergic responses of individual MSNs through activation of NK1 receptors. $\boldsymbol{A}$, Typical positioning of the patch electrode used for single whole-cell recordings, and of the stimulating electrodes used to elicit orthodromic activation of corticostriatal fibers and antidromic activation of MSN axons in sagittal slices. Corticostriatal fibers were preferentially stimulated with an electrode placed in the $\mathrm{CC}$, between the cortex and the striatum (area 1). MSN axons were stimulated with an electrode placed in the GP (area 2). $\boldsymbol{B}$, Simplified diagram illustrating the neurons and axons activated in these experiments. Antidromic spikes travel from the GP to the striatum, invading MSN axon collaterals and releasing neurotransmitters, including substance $P$, in the striatum. Corticostriatal fibers impinge on the recorded MSN, eliciting glutamatergic responses. $\boldsymbol{C}$, In a representative experiment, preceding GP stimulation strongly increased $(p<0.001)$ the amplitude of CC-evoked responses in a MSN. Five stimuli were delivered at 100 $\mathrm{Hz}$ in the GP, and were followed by a single stimulation in the CC; the interval between the first GP stimulus and the CC stimulus was $500 \mathrm{~ms}$. This protocol was alternated (every $10 \mathrm{~s}$ ) with one in which a single stimulus was delivered in the CC without preceding GP stimuli. Each trace is the average of 150 consecutive presentations of a certain protocol. Superimposed traces on the right show the average evoked EPSP with (gray) and without (black) preceding GP stimulation. Subsequent bath application of L-732,138 fully abolished the effects of GP stimulation on corticostriatal responses. L-732,138 also caused a significant decrease in the amplitude of control corticostriatal responses. Traces are the average of 100 consecutive presentations. D, Cumulative distribution of CC-evoked EPSP amplitude for the MSN of $\boldsymbol{C}$, in the absence (black line) or presence (gray line) of preceding GP stimulation, before (left) and after (right) application of $L-732,138$. In the absence, but not in the presence, of $L-732,138$, preceding GP stimulation causes a substantial rightward shift of the cumulative distribution of EPSP amplitude.

compared. In 8 experiments, single CC stimuli were delivered every $10 \mathrm{~s}$, and were preceded sequentially by (1) no GP stimulation; (2) GP stimulation at $250 \mathrm{~ms}$ interval; and (3) GP stimulation at $500 \mathrm{~ms}$ interval. In 6 of these experiments GP stimulation caused significant $(p<0.001)$ facilitation both at 250 and $500 \mathrm{~ms}$ intervals; in the 2 remaining experiments no significant facilitation was observed at either interval. In all cases where there was facilitation, the effects were significantly $(p<0.05)$ larger at 250 $\mathrm{ms}$ than at $500 \mathrm{~ms}$. An example of this phenomenon is illustrated in Figure 7A. The average effects observed in each of these 6 experiments for 250 and $500 \mathrm{~ms}$ intervals are shown in the plot of Figure $7 B$. 
A

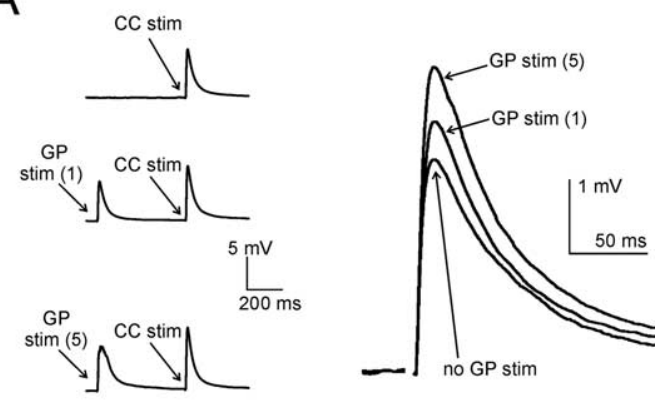

B

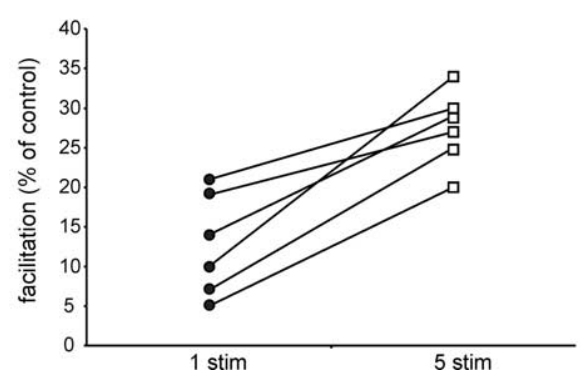

Figure 6. Five GP stimuli are more effective than one at facilitating (C-evoked responses. $A$, In this representative experiment, a single CC stimulus (delivered every $10 \mathrm{~s}$ ) was sequentially preceded by (1) no GP stimulation; (2) one GP stimulus (preceding (C stimulation by $500 \mathrm{~ms}$ ); and (3) five GP stimuli (also preceding (C stimulation by $500 \mathrm{ms).} \mathrm{Traces} \mathrm{are} \mathrm{averages} \mathrm{of} 90$ consecutive presentations of each protocol. Average C(-evoked responses for the three conditions are enlarged and superimposed on the right. While both one and five preceding GP stimuli significantly facilitate $\mathrm{CC}$-evoked responses, facilitation is significantly larger with five preceding stimuli than with a single one. $\boldsymbol{B}$, Similar results were found in each of six different experiments. For each experiment, data points representing average facilitation of $\mathrm{CC}$-evoked responses in the presence of one or five preceding GP stimuli are connected by a line.

In a different series of experiments, we used a similar protocol to compare the effects of 500 and $1000 \mathrm{~ms}$ intervals. Among the MSNs tested with this protocol, significant $(p<0.001)$ facilitatory effects were observed in $7 / 14$ cases at $500 \mathrm{~ms}$ intervals (in the other 7 no effects were present at either 500 or $1000 \mathrm{~ms}$ ). In 3 of the 7 cases in which significant effects were present at $500 \mathrm{~ms}$, significant $(p<0.05)$ facilitation was also observed for $1000 \mathrm{~ms}$ intervals, although in all cases this facilitation was significantly $(p<0.05)$ smaller than at $500 \mathrm{~ms}$ intervals. In the remaining 4 MSNs in which significant effects were present at $500 \mathrm{~ms}$, no significant facilitation was observed at $1000 \mathrm{~ms}$. These results are illustrated in Figure 7C.

The overall distribution of the effects of GP stimulation on cortical responses for 250, 500, and $1000 \mathrm{~ms}$ in all MSNs tested (including those in which no significant effects were observed) is presented in the histograms of Figure $8 \mathrm{~A}$. Longer intervals ( 2 and $3 \mathrm{~s} ; n=7$ and $n=6$, respectively) were also tested in different experiments, in which significant facilitation was present at 500 $\mathrm{ms}$ intervals. However, no significant facilitation was observed in any of these cases. The average facilitation of CC-evoked responses as a function of the interval between GP and CC stimulation are plotted in Figure $8 B$; these data include all experiments in which significant facilitatory effects were observed for at least one interval. This plot shows that after GP stimulation there is a an approximately exponential decay of the facilitatory effects, which are maximal at the shortest interval tested $(250 \mathrm{~ms})$, and disappear after 1-2 s.

To test whether synaptically released SP actually increased the
A
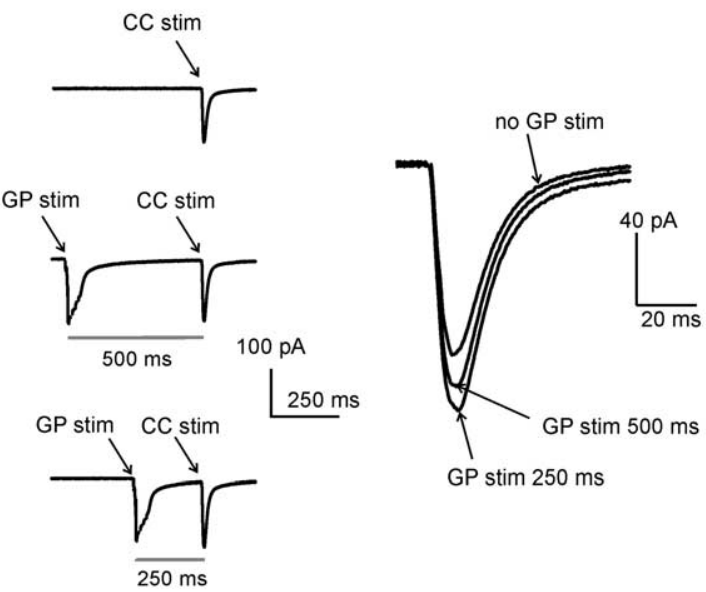

B
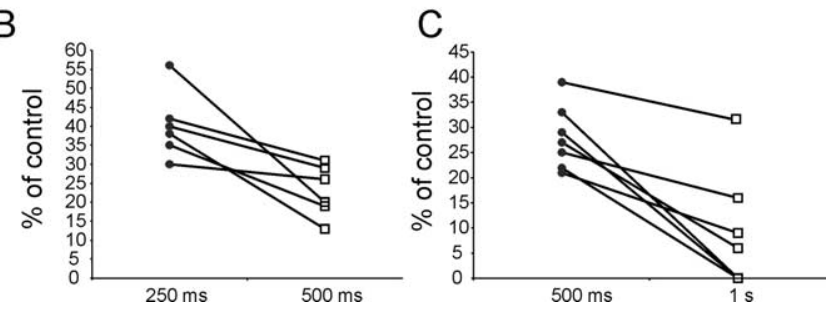

Figure 7. Facilitation of corticostriatal responses induced by GP stimulation is larger at 250 ms than at 500 ms intervals. $A$, In this representative voltage-clamp experiments ( $V_{\text {holding }}=$ $-80 \mathrm{mV}$ ), a single (C stimulus was delivered every $10 \mathrm{~s}$. These stimuli were sequentially preceded by (1) no GP stimulation; (2) five GP stimuli preceding CC stimulation by $500 \mathrm{~ms}$; and (3) five GP stimuli preceding CC stimulation by $250 \mathrm{~ms}$. Traces are averages of 120 consecutive repetitions for each protocol. Average synaptic currents evoked by CC stimulation for each of these three conditions are enlarged and superimposed on the right. While at both intervals GP stimuli significantly ( $p<0.001)$ facilitated CC-evoked responses, facilitation was significantly $(p<0.05)$ larger when the GP stimuli preceded C stimulation by $250 \mathrm{~ms}$ than by $500 \mathrm{~ms}$. $\boldsymbol{B}$, Similar results were found in six different experiments. For each experiment, data points representing average facilitation of $\mathrm{CC}$-evoked responses in the presence of GP stimuli preceding $C \mathrm{C}$ stimulation by 250 and $500 \mathrm{~ms}$ are connected by a line. $C$, In a different series of experiments, a similar protocol was used to compare the effects of GP stimuli preceding CC stimulation by 500 and $1000 \mathrm{~ms}$. In each of the seven cases represented in the plot, significant $(p<0.01)$ facilitation was observed at 500 ms intervals. In three of these cases no facilitation was present at $1000 \mathrm{~ms}$ intervals; in the remaining four cases, there was significant $(p<0.05)$ facilitation of CC-evoked responses at 1000 ms intervals, but this was always significantly $(p<0.05)$ smaller than at 500 ms intervals.

probability of action potential generation in MSNs, we performed a series of experiments using higher CC stimulation intensities, so that the evoked EPSPs were large enough to drive the MSN membrane potential close to spike threshold. We set the CC stimulation intensity at a level which evoked spikes in $25-40 \%$ of cases (without preceding GP stimuli). After this, we applied a protocol similar to that used to test the effects of GP stimulation on the smaller subthreshold CC-evoked responses: a single CC stimulus was applied every $10 \mathrm{~s}$; every other CC stimulus was preceded (by $500 \mathrm{~ms}$ ) by a burst of $5 \mathrm{GP}$ stimuli at $100 \mathrm{~Hz}$. At least 200 consecutive CC stimuli were delivered in each pharmacological condition. The number of spikes elicited in the absence or in the presence of preceding GP stimuli were then statistically compared using McNemar's test for paired data. In 7/8 MSNs, the probability of observing an action potential was strongly and significantly $(p<0.05)$ larger when GP stimuli preceded the CC stimulation. On average, spikes were elicited by $28 \pm 6 \%$ of CCevoked EPSPs not preceded by GP stimuli, and by $58 \pm 5 \%$ of CC-evoked EPSPs preceded by GP stimuli. L-732,138 was subse- 


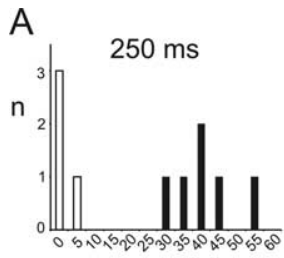

$\%$ potentiation

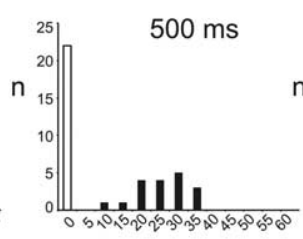

$\%$ potentiation

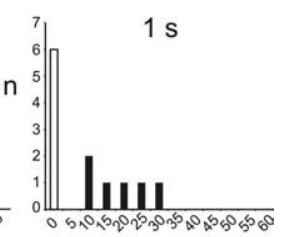

$\%$ potentiation
B

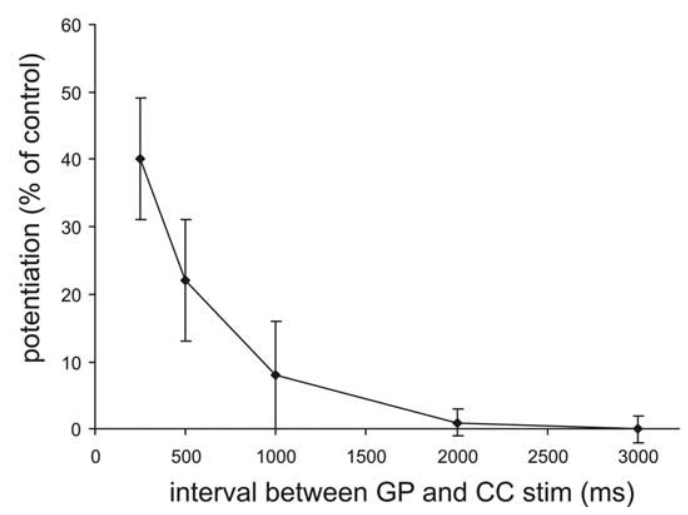

Figure 8. Time course of facilitatory effects of GP stimulation on CC-evoked responses. $\boldsymbol{A}$, These histograms illustrate the overall distribution of the effects of five preceding GP stimuli on CC-evoked responses for intervals of 250,500 , and $1000 \mathrm{~ms}$. While a similar percentage of cases in which no significant facilitation was present (white columns) is found for all intervals, in cases where significant ( $p<0.05$ ) facilitation is present (black columns) its extent is larger for 250 ms than for $500 \mathrm{~ms}$, and for $500 \mathrm{~ms}$ than for $1000 \mathrm{~ms}$ intervals. $\boldsymbol{B}$, Average effects of GP stimulation on CC-evoked responses as a function of the interval preceding CC stimulation. Data from all experiments in which significant effects were found for at least one interval are included. Effects are maximal for the shortest interval $(250 \mathrm{~ms})$, and decay in an approximately exponential manner. Error bars represent SDs.

quently applied in each of these experiments; since L-732,138 per se caused a decrease in the amplitude of evoked glutamatergic responses (Blomeley and Bracci, 2008), the CC stimulation intensity was slightly increased so that CC-evoked responses elicited spikes in 25-35\% of cases. Under these conditions, the ability of preceding GP stimuli to increase spike generation probability was completely abolished, and the numbers of spikes evoked with or without preceding GP stimuli were not significantly different. A representative example of these experiments is illustrated in Figure 9. We concluded that the facilitatory effects of synaptically released SP increased the probability of MSN spike generation, and were therefore functionally excitatory.

\section{Discussion}

This is the first demonstration that striatal projection neurons interact in an excitatory manner; we showed that action potentials in MSNs can facilitate glutamatergic responses and increase the probability of action potential generation in neighboring MSNs with a rapid time course, and that these effects are entirely mediated by NK1 receptors. These results are particularly significant as MSNs are traditionally considered to form a network of mutually inhibiting GABAergic units, and several theories of striatal function are based on this feature and its ability to create competitive dynamics (Plenz, 2003; Wickens et al., 2007; Wilson, 2007). The present data suggest that cooperative dynamics also exist between MSNs, and this may have profound implications for our understanding of the striatal operation.

Using paired recordings and antidromic stimulation of MSN axons, we were able to investigate in detail the extent and time
A

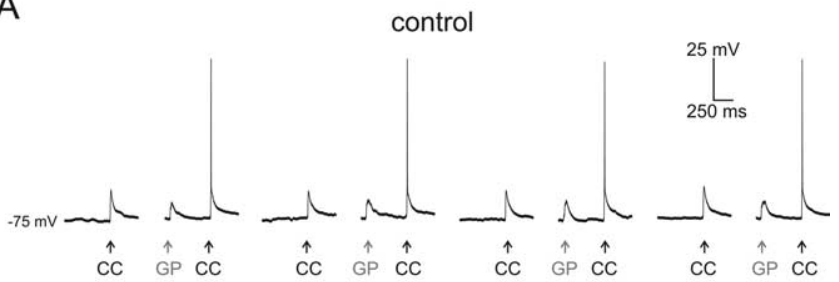

L-732,138

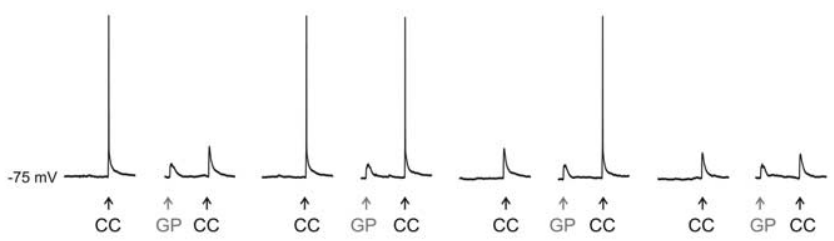

B

control

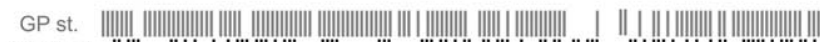
no GP st. $\||||||||||||||||||||||||||||||||||||||||||||||||||||||||||||||||||| \mid$

\begin{tabular}{|c|c|c|c|c|c|c|}
\hline & & $1 T$ & \begin{tabular}{l|l}
-1 & 1
\end{tabular} & 1,1 & 1 & 1 \\
\hline 0 & 500 & 1000 & $\begin{array}{c}1500 \\
\text { time (s) }\end{array}$ & 2000 & 2500 & 3000 \\
\hline
\end{tabular}

L-732, 138

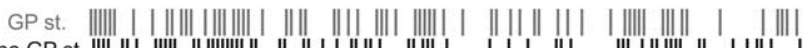

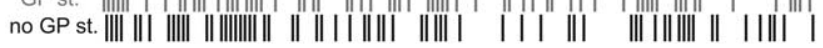

\begin{tabular}{|c|c|c|c|c|c|c|}
\hline & 11 & $T 1$ & $T$ & $T$ & 11 & $T$ \\
\hline 0 & 500 & 1000 & $\begin{array}{c}1500 \\
\text { time }(\mathrm{s})\end{array}$ & 2000 & 2500 & 3000 \\
\hline
\end{tabular}

Figure 9. Antidromic MSN stimulation facilitates spike generation through NK1 receptor activation. $A$, Glutamatergic responses were evoked in a MSN by a single CC stimulation every 10 seconds; every other CC stimulation was preceded by five GP stimuli (at $100 \mathrm{~Hz}$ ). Eight consecutive $C C$ responses (four of which preceded by GP stimuli) are shown in the absence (upper traces) and in the presence (lower traces) of $L-732,138$. In the absence, but not in the presence, of L-732,138, CC stimulation evoked a spike more frequently when it was preceded by GP stimuli. Stimulation intensity was increased in the presence of L-732,138, which had caused a slight decrease in CC-evoked response amplitude. $\boldsymbol{B}$, Raster plots for the experiment illustrated in $A$. A black vertical dash indicate that a spike was elicited by CC stimulation not preceded by GP stimuli, while a gray vertical dash indicate that a spike was elicited by CC stimulation preceded by GP stimuli. Three hundred six CC stimuli were delivered in the absence of L-732,138, and 300 CC stimuli were delivered in the presence of L-732,138.

course of these facilitatory effects. Glutamatergic responses were evoked in MSNs by stimuli delivered in (or close to) the white matter between the cortex and the striatum; this is likely to activate corticostriatal axons, although involvement of thalamostriatal fibers (Smeal et al., 2007) is also likely, especially in coronal slices in which stimulation current has to spread further to activate glutamatergic fibers impinging on MSNs. In this case, stimulation is also likely to activate local striatal fibers and neurons, in addition to glutamatergic axons; however, the effects of this activation were minimal, because all the experiments were performed in the presence of antagonists for GABA, opioid, and cholinergic receptors. $\mathrm{D}_{1}$ and $\mathrm{D}_{2}$ dopamine receptors were also blocked, so that their involvement in the present phenomena can be ruled out.

In connected pairs, when a burst of five spikes was evoked in a MSN, maximal effects (on average 14\% facilitation) were present $100 \mathrm{~ms}$ after the first spike of the burst, while no facilitation was 
present after $50 \mathrm{~ms}$, and little residual facilitation was found after $200 \mathrm{~ms}$. The facilitatory effects elicited by five antidromic stimuli were larger and longer lasting, presumably because activation of a population of MSNs caused a much larger release of SP than that of a single MSN. For technical reasons, with antidromic stimulation we were not able to explore intervals smaller than $250 \mathrm{~ms}$; however, we observed maximal effects $250 \mathrm{~ms}$ after the first GP stimulus (on average $40 \%$ facilitation), and robust effects persisting after $500 \mathrm{~ms}$ (on average $22 \%$ facilitation). When the data from the two sets of experiments are combined, the picture emerges that NK1 receptor-mediated facilitatory effects would peak $\sim 100 \mathrm{~ms}$ after the first spike of a short burst, and decay in an approximately exponential manner over 1-2 s. In interpreting these results, one should consider that these experiments were performed at room temperature, and it is plausible that this phenomenon may have a faster time course at physiological temperature.

We did not explore systematically which pattern of action potentials produced maximal facilitatory effects, but antidromic stimulation experiments showed that the facilitatory effects of a burst of 5 stimuli were invariably large than those of a single one. In vivo intracellular recordings from MSNs have shown that these cells often fire bursts of 3-10 action potentials at frequencies similar to those used in this study $(77-100 \mathrm{~Hz})$ during up states (Stern et al., 1997; Sachdev et al., 2004); based on present results, this pattern of activity would be well suited to cause robust facilitatory effects in connected MSNs.

Facilitation of glutamatergic inputs was present (either in one direction or bidirectionally) in 23\% of MSN pairs tested; this connectivity rate is similar to the one found for direct GABAergic synapses (Tunstall et al., 2002; Koos et al., 2004; Shindou et al., 2008); we never observed spike-induced inhibition of glutamatergic inputs to MSNs. It should be noted, however, that this could be due to the fact that $\mathrm{GABA}_{\mathrm{B}}$ and opioid receptors, which may be activated by neurotransmitters released by MSNs, and mediate presynaptic inhibition (Calabresi et al., 1992; Jiang and North, 1992; Miura et al., 2008), were pharmacologically blocked. Since $\mathrm{GABA}_{\mathrm{A}}$ receptors were also pharmacologically blocked, we could not determine whether postsynaptic GABAergic connections and SPmediated presynaptic facilitation coexisted in the same pairs, or were substantially segregated. This is undoubtedly an important issue that deserves further investigation. The distribution and the extent of SP-mediated effects in preweaning and postweaning $(>\mathrm{P} 21)$ animals were similar, suggesting that this was not a transient developmental phenomenon taking place before the first three postnatal weeks.

While it was not possible to demonstrate directly that facilitation of glutamatergic input by endogenous SP was mediated by presynaptic receptors, results from previous studies strongly supports this hypothesis. We recently found that exogenous SP facilitates glutamatergic responses of MSNs through a presynaptic mechanism (Blomeley and Bracci, 2008). Furthermore, anatomical studies have showed that presynaptic NK1 receptors are present on corticostriatal and thalamostriatal glutamatergic axonal terminals impinging on MSNs in the rat and the monkey (Jakab and Goldman-Rakic, 1996). How did SP reach these presynaptic receptors? SP-immunoreactive axon terminals arising from MSNs form synapses with spines and dendritic shafts of other MSNs (Bolam and Izzo, 1988; Yung et al., 1996). Corticostriatal terminals synapse on dendritic spines, and thalamostriatal terminals on dendritic shafts (Jakab and Goldman-Rakic, 1996). Thus, diffusion may allow synaptically released SP to reach
NK1 receptors located on glutamatergic terminals, as originally hypothesized by Jakab and Goldman-Rakic (1996).

In this study, we did not directly identify the subpopulation of the recorded MSNs; however, the MSNs whose action potentials caused facilitation of glutamatergic input are most likely able to release SP, and therefore belong to the "direct pathway" subpopulation, which also express $\mathrm{D}_{1}$ dopamine receptor. The subpopulation of the MSNs whose glutamatergic inputs were facilitated is less easily identified. In pairs in which reciprocal facilitation of glutamatergic inputs was present, both MSNs must have been able to release SP; glutamatergic inputs into these cells were facilitated. We can therefore exclude that the MSNs that had their input facilitated belonged exclusively to the "indirect pathway" subpopulation. On the other hand, anatomical studies have shown that SP-immunoreactive terminals form synapses on the dendrites of both $\mathrm{D}_{1}$ and $\mathrm{D}_{2}$ receptor bearing MSNs (Yung et al., 1996). These data suggest that SP-mediated facilitation of glutamatergic input was not segregated to one of the two MSN subpopulations. A diagram illustrating the possible synaptic arrangement underlying SP-mediated interactions between MSNs is presented in supplemental Figure 1 (available at www.jneurosci.org as supplemental material).

While some of the effects of endogenously released SP overlap with those previously observed with exogenous SP application (Blomeley and Bracci, 2008), there are also some important differences. Exogenous SP facilitated evoked glutamate responses acting on both $\mathrm{NK} 1$ and $\mathrm{NK} 3$ receptors. Conversely, the facilitatory effects of MSN action potentials were always completely blocked by NK1 receptor antagonist, showing that NK3 receptors were not significantly activated by endogenous SP. This may be due to a different localization of NK3 receptors on glutamatergic terminals and/or to their lower affinity for SP compared with NK1 receptors (Almeida et al., 2004). Exogenous SP also caused direct depolarizing effects in MSNs through activation of postsynaptic NK1 receptors (possibly a short isoform not detected in some immunohistochemical studies), causing an increase in inwardrectifying chloride conductances (Blomeley and Bracci, 2008). However, MSN action potentials did not cause detectable postsynaptic effects in neighboring MSNs; in these experiments either a single MSN, or a population of MSNs generated bursts of five spikes every 10-30 s for long periods of time (often $>60 \mathrm{~min}$ ). The absence of fast or slow responses in MSNs suggests that postsynaptic NK1 receptors were not activated by endogenous SP, perhaps as a result of their extrasynaptic location. These observations highlight the importance of studying the effects of presynaptic and postsynaptic receptor activation by endogenous transmitters released by controlled patterns of action potentials, as the spatiotemporal distribution of this release can strongly differ from that of exogenous ligand applications.

SP-mediated facilitation of glutamatergic transmission was observed here in the presence of opioid and $\mathrm{GABA}_{\mathrm{B}}$ receptor antagonists. Enkephalin, dynorphin, and GABA, are also released by MSNs (Gerfen, 1992), and may activate presynaptic receptors located on glutamatergic terminals; therefore, in vivo MSNs may also inhibit glutamate release through activation of $\mathrm{GABA}_{\mathrm{B}}$ and opioid receptors (Calabresi et al., 1992; Jiang and North, 1992). The net effects on glutamatergic transmission will depend on the distribution of these receptors on the glutamatergic terminals and the ability of the neurotransmitters released by MSNs to reach these receptors.

The dense local network of MSN axon collaterals is a salient anatomical feature of the striatum, and direct synaptic com- 
munication among MSNs has been extensively investigated (Tunstall et al., 2002; Koos et al., 2004). One controversial issue has been whether GABAergic inhibition between MSNs is strong enough to significantly shape striatal dynamics (Wickens et al., 2007; Wilson, 2007). The magnitude of SPmediated facilitation of glutamatergic responses found in the present study was substantial; action potentials in a single MSN increased the amplitude of the glutamatergic responses in a connected MSN typically by $10-20 \%$, and facilitation as large as $30-50 \%$ was caused by antidromic stimulation of MSN axons. While the latter protocol may induce an artificial degree of synchronicity in MSN firing, these cells do receive strongly correlated cortical inputs in vivo (Stern et al., 1998); thus, large, semisynchronous SP release may occur in the intact brain, causing strong facilitation of glutamatergic inputs in a set of MSNs. The striatal output, as well as the formation of long-term corticostriatal memories (Gubellini et al., 2004; Calabresi et al., 2007), will be determined, for a certain barrage of cortical inputs, by a dynamic balance between SP-mediated facilitation of glutamatergic inputs, and feedforward and feedback GABAergic inhibition (Tepper et al., 2008). Unraveling the synaptic architecture of presynaptic and postsynaptic connections between striatal neurons will be critical to understand the operation of this nucleus and its role in motor control.

\section{References}

Albin RL, Young AB, Penney JB (1995) The functional anatomy of disorders of the basal ganglia. Trends Neurosci 18:63-64.

Alexander GE, Crutcher MD (1990) Functional architecture of basal ganglia circuits: neural substrates of parallel processing. Trends Neurosci 13:266-271.

Almeida TA, Rojo J, Nieto PM, Pinto FM, Hernandez M, Martín JD, Candenas ML (2004) Tachykinins and tachykinin receptors: structure and activity relationships. Curr Med Chem 11:2045-2081.

Baranauskas G, Traversa U, Rosati AM, Nistri A (1995) An NK1 receptordependent component of the slow excitation recorded intracellularly from rat motoneurons following dorsal root stimulation. Eur J Neurosci $7: 2409-2417$.

Bar-Gad I, Morris G, Bergman H (2003) Information processing, dimensionality reduction and reinforcement learning in the basal ganglia. Prog Neurobiol 71:439-473.

Beiser DG, Houk JC (1998) Model of cortical-basal ganglionic processing: encoding the serial order of sensory events. J Neurophysiol 79:3168-3188.

Blomeley C, Bracci E (2008) Substance P depolarizes striatal projection neurons and facilitates their glutamatergic inputs. J Physiol 586:2143-2155.

Bolam JP, Izzo PN (1988) The postsynaptic targets of substance $\mathrm{P}$-immunoreactive terminals in the rat neostriatum with particular reference to identified spiny striatonigral neurons. Exp Brain Res 70:361-377.

Bolam JP, Hanley JJ, Booth PA, Bevan MD (2000) Synaptic organisation of the basal ganglia. J Anat 196:527-542.

Bracci E, Centonze D, Bernardi G, Calabresi P (2004) Engagement of rat striatal neurons by cortical epileptiform activity investigated with paired recordings. J Neurophysiol 92:2725-2737.

Calabresi P, Mercuri NB, Stefani A, Bernardi G (1992) Physiological role of GABAb receptors in the mammalian neostriatum. Adv Biochem Psychopharmacol 47:217-221.

Calabresi P, Picconi B, Tozzi A, Di Filippo M (2007) Dopamine-mediated regulation of corticostriatal synaptic plasticity. Trends Neurosci $30: 211-219$.

Gerfen CR (1992) The neostriatal mosaic: multiple levels of compartmental organization in the basal ganglia. Annu Rev Neurosci 15:285-320.

Gertler TS, Chan CS, Surmeier DJ (2008) Dichotomous anatomical properties of adult striatal medium spiny neurons. J Neurosci 28:10814-10824.
Graveland GA, DiFiglia M (1985) The frequency and distribution of medium-sized neurons with indented nuclei in the primate and rodent neostriatum. Brain Res 327:307-311.

Graybiel AM, Aosaki T, Flaherty AW, Kimura M (1994) The basal ganglia and adaptive motor control. Science 265:1826-1831.

Gubellini P, Pisani A, Centonze D, Bernardi G, Calabresi P (2004) Metabotropic glutamate receptors and striatal synaptic plasticity: implications for neurological diseases. Prog Neurobiol 74:271-300.

Gurney K, Prescott TJ, Wickens JR, Redgrave P (2004) Computational models of the basal ganglia: from robots to membranes. Trends Neurosci 27:453-459.

Guzmán JN, Hernández A, Galarraga E, Tapia D, Laville A, Vergara R, Aceves J, Bargas J (2003) Dopaminergic modulation of axon collaterals interconnecting spiny neurons of the rat striatum. J Neurosci 23:8931-8940.

Jaeger D, Kita H, Wilson CJ (1994) Surround inhibition among projection neurons is weak or nonexistent in the rat neostriatum. J Neurophysiol 72:2555-2558.

Jakab RL, Goldman-Rakic P (1996) Presynaptic and postsynaptic subcellular localization of substance P receptor immunoreactivity in the neostriatum of the rat and rhesus monkey (Macaca mulatta). J Comp Neurol 369:125-136.

Jiang ZG, North RA (1992) Pre- and postsynaptic inhibition by opioids in rat striatum. J Neurosci 12:356-361.

Kincaid AE, Zheng T, Wilson CJ (1998) Connectivity and convergence of single corticostriatal axons. J Neurosci 18:4722-4731.

Kita T, Kita H, Kitai ST (1984) Passive electrical membrane properties of rat neostriatal neurons in an in vitro slice preparation. Brain Res 300:129-139.

Koos T, Tepper JM, Wilson CJ (2004) Comparison of IPSCs evoked by spiny and fast-spiking neurons in the neostriatum. J Neurosci 24:7916-7922.

Miura M, Masuda M, Aosaki T (2008) Roles of mu-opioid receptors in GABAergic synaptic transmission in the striosome and matrix compartments of the striatum. Mol Neurobiol 37:104-115.

Nisenbaum ES, Wilson CJ (1995) Potassium currents responsible for inward and outward rectification in rat neostriatal spiny projection neurons. J Neurosci 15:4449-4463.

Pakhotin P, Bracci E (2007) Cholinergic interneurons control the excitatory input to the striatum. J Neurosci 27:391-400.

Plenz D (2003) When inhibition goes incognito: feedback interaction between spiny projection neurons in striatal function. Trends Neurosci 26:436-443.

Redgrave P, Gurney K (2006) The short-latency dopamine signal: a role in discovering novel actions? Nat Rev Neurosci 7:967-975.

Sachdev RN, Ebner FF, Wilson CJ (2004) Effect of subthreshold up and down states on the whisker-evoked response in somatosensory cortex. J Neurophysiol 92:3511-3521.

Shindou T, Arbuthnott GW, Wickens JR (2008) Actions of adenosine A2A receptors on synaptic connections of spiny projection neurons in the neostriatal inhibitory network. J Neurophysiol 99:1884-1889.

Smeal RM, Gaspar RC, Keefe KA, Wilcox KS (2007) A rat brain slice preparation for characterizing both thalamostriatal and corticostriatal afferents. J Neurosci Methods 159:224-235.

Somogyi P, Bolam JP, Smith AD (1981) Monosynaptic cortical input and local axon collaterals of identified striatonigral neurons. A light and electron microscopic study using the Golgi-peroxidase transportdegeneration procedure. J Comp Neurol 195:567-584.

Stern EA, Kincaid AE, Wilson CJ (1997) Spontaneous subthreshold membrane potential fluctuations and action potential variability of rat corticostriatal and striatal neurons in vivo. J Neurophysiol 77:1697-1715.

Stern EA, Jaeger D, Wilson CJ (1998) Membrane potential synchrony of simultaneously recorded striatal spiny neurons in vivo. Nature 394:475-478.

Tecuapetla F, Carrillo-Reid L, Guzmán JN, Galarraga E, Bargas J (2005) Different inhibitory inputs onto neostriatal projection neurons as revealed by field stimulation. J Neurophysiol 93:1119-1126.

Tepper JM, Koós T, Wilson CJ (2004) GABAergic microcircuits in the neostriatum. Trends Neurosci 27:662-669.

Tepper JM, Wilson CJ, Koós T (2008) Feedforward and feedback inhibition in neostriatal GABAergic spiny neurons. Brain Res Rev 58:272-281. 
Testa CM, Standaert DG, Landwehrmeyer GB, Penney JB Jr, Young AB (1995) Differential expression of mGluR5 metabotropic glutamate receptor mRNA by rat striatal neurons. J Comp Neurol 354:241-252.

Tunstall MJ, Oorschot DE, Kean A, Wickens JR (2002) Inhibitory interactions between spiny projection neurons in the rat striatum. J Neurophysiol 88:1263-1269.

Wang Z, Kai L, Day M, Ronesi J, Yin HH, Ding J, Tkatch T, Lovinger DM, Surmeier DJ (2006) Dopaminergic control of corticostriatal long-term synaptic depression in medium spiny neurons is mediated by cholinergic interneurons. Neuron 50:443-452.

Wickens JR, Arbuthnott GW, Shindou T (2007) Simulation of GABA function in the basal ganglia: computational models of GABAergic mechanisms in basal ganglia function. Prog Brain Res 160:313-329.
Wilson CJ (1987) Morphology and synaptic connections of crossed corticostriatal neurons in the rat. J Comp Neurol 263:567-580.

Wilson CJ (2007) GABAergic inhibition in the neostriatum. Prog Brain Res 160:91-110.

Wilson CJ, Groves PM (1980) Fine structure and synaptic connections of the common spiny neuron of the rat neostriatum: a study employing intracellular inject of horseradish peroxidase. J Comp Neurol 194:599-615.

Wilson CJ, Chang HT, Kitai ST (1983) Origins of post synaptic potentials evoked in spiny neostriatal projection neurons by thalamic stimulation in the rat. Exp Brain Res 51:217-226.

Yung KK, Smith AD, Levey AI, Bolam JP (1996) Synaptic connections between spiny neurons of the direct and indirect pathways in the neostriatum of the rat: evidence from dopamine receptor and neuropeptide immunostaining. Eur J Neurosci 8:861-869. 